\title{
ВЫБОР ОПТИМАЛЬНОГО КОЛИЧЕСТВА КОТЛОАГРЕГАТОВ ПРИ РЕКОНСТРУКЦИИ ИСТОЧНИКА ТЕПЛОСНАБЖЕНИЯ
}

\author{
Збараз Леонид Иосифович', \\ Zbaraz_Len@ukr.net \\ Чичерин Станислав Викторович², \\ man_csv@hotmail.com

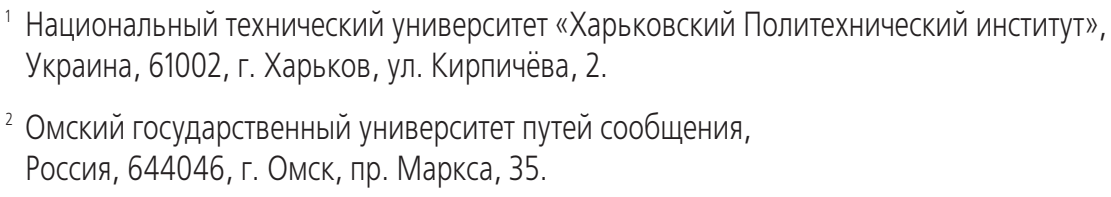

Актуальность. Реконструкция источника теплоснабжения может привести к появлению чрезмерного резерва мощности, что сделает его эксплуатацию дорогостоящей из-за больших потерь в периоды низкой тепловой нагрузки и значительных капиталовложений. С другой стороны, энергоэффективное производство и преобразование энергии на основе георесурсов не всегда возможно в периоды максимального потребления тепловой энергии. Чтобы обеспечить такой спрос, как правило, создаются пиковые элементы генерации. Поскольку такие источники обычно маломощны и предназначены для краткосрочной работы, в качестве топлива часто предусматривается дорогостоящее топливо, что делает задачу оптимального конфигурирования оборудования при реконструкции источника теплоснабжения актуальной.

Цель и задачи исследования. Формализовано цель можно выразить следующим образом: среди всех возможных вариантов установки оборудования выбрать такой, при котором прямые затраты энергоносителей за отопительный сезон будут минимальными, но при этом заданная тепловая нагрузка будет обеспечиваться.

Методы. Оптимальная схема выбиралась после расчета нескольких конфигураций оборудования котельной и сравнения прямых затрат за отопительный сезон для разных климатических зон. Ограничения, наложенные на единичную величину вырабатываемой мощности и КПД, и принятая методика расчета привели к минимально возможному количеству независимых переменных. Общая тепловая нагрузка распределялась между несколькими котлами, что, исходя из практики эксплуатации, является предпочтительным вариантом, когда агрегаты связаны между собой по параллельной схеме. Принята схема, когда дополнительный котел, находящийся в резерве, включается, если заданная нагрузка не может быть покрыта котлами, уже находящимися в работе, о чем сигнализируют показания расходомера пара или датчик температуры теплоносителя в подающем трубопроводе. Поскольку зависимость КПД котла от удельной нагрузки на стадии проектирования и реконструкции неизвестна, для расчётов принимаем среднестатистическую зависимостью КПД брутто котла от удельной нагрузки. Решать задачу предлагается с помощью средств автоматизации расчетов, например, прикладного пакета программ Statistika 6.

Результаты. Были выполнены расчёты для котельных, расположенных в Республике Татарстан, Харьковской и Омской областях. Принятая тарифная составляющая достигала 3,68 руб/кВт•ч электрической энергии и 5,48 руб. за кубометр природного газа. Для подключённой тепловой нагрузки 4,2 МВт, рассматривая одну из шести комбинаций компоновки: от двух котлов одинаковой мощности по 2,5 МВт каждый до набора котельного оборудования 2,5 МВт, 1,6 МВт и 1,0 МВт, было установлено, что ситуация для различных объектов принципиально отличается. Для Харьковской и Омской областей, отличающихся наиболее теплым и холодным климатом, соответственно, оптимальным является вариант установки котлов мощностью 2,5, 1,6 и 1,0 МВт, а для г. Казани - два котла по 2,0 и один - 1,0 МВт.

Выводы. Использованный в настоящей работе метод решения двухуровневой оптимизационной задачи, для решения которой применялся принцип декомпозиции, позволил установить оптимальную из шести возможных комбинаций компоновку котельной. Разработанный подход может стать полезным инструментом в руках проектировщика. Полученные результаты интересны и определенно должны подтвердиться на практике. В частности, показана возможность производства при минимальных затратах первичных энергоносителей, что в свою очередь приведет к снижению вредного воздействия на окружающую среду и позволит говорить об энергоэффективной технологии производства и преобразования энергии на основе георесурсов.

\section{Ключевые слова:}

Энергоносители, теплоснабжение, природный газ, котельная, производство,

генерация, оптимизация, минимизация, затраты.

\section{Введение}

В настоящий момент большое количество средств вкладывается в модернизацию источников теплоснабжения. Целью реконструкции является не только повышение надёжности, но и снижение прямых затрат на выработку тепловой энергии и отпуск её потребителям. Экономичность работы источника, в свою очередь, может быть достигнута при эксплуатации оборудования в диапазоне максимального КПД.
Энергоэффективное производство и преобразование энергии на основе георесурсов является темой работы многих ученых на протяжении длительного периода времени. В то же время большая часть работ связана с использованием нетрадиционных и возобновляемых источников энергии (НВИЭ), например [1-3]. Широкое применение последних возможно, главным образом, в странах Западной Европы. В России и Украине, где не имеется полноценной нормативной базы, описывающей 
строительство и подключение таких источников к сетям общего пользования, а вопросы сбыта произведенной энергии не отрегулированы, перечисленные работы не являются актуальными в краткосрочной перспективе.

Между тем энергоэффективное производство на основе георесурсов хорошо согласуется с тезисами, выдвинутыми в [4], в числе которых реконструкция промышленно-отопительных котельных и более широкое внимание к НВИЭ. Традиционные котельные установки, использующие в качестве топлива природный газ, описаны в [5], однако большую часть объема материала занимает эксергетический анализ уже имеющегося оборудования без учета возможной модернизации или реконструкции.

C точки зрения решения задачи оптимизации (минимизации составляющих) наиболее близки и полезны статьи $[6,7]$, но если в первой работе акцент сделан не столько на затратной части, сколько на удовлетворении спроса на тепловую энергию, вырабатываемую несколькими источниками теплоснабжения, работающими на общую тепловую сеть, то во второй много внимания уделяется эксплуатации оборудования, причем в качестве источника рассматривается только ТЭЦ. Комбинированная выработка в условиях современной рыночной экономики и политико-экономической формации исследована в [8]. Если абстрагироваться от теплофикации, обращает на себя внимание статья [9], где изучена реконструкция объекта районной генерации (квартальной тепловой станции, РТС), количество последних, например, в г. Москва достигает 68.

Модернизация источника теплоснабжения тема исследования [10], однако рассматривается сложный и дорогостоящий процесс замены твердотопливных котлов на газотурбинные установки малой мощности, требующий многомиллионных инвестиций. Котлы, где также в качестве первичного энергоносителя применяются угли, подробно исследованы в [11]. Моделирование пылеугольного котла Кировской ТЭЦ-4 [12] позволило выявить ряд мер, при реализации которых возможно повышение режимных характеристик и достижение экономии топлива, т. е. решаются задачи, поставленные и в настоящей работе.

Что касается конфигурировании генерирующего оборудования, то с некоторой оговоркой результаты настоящего исследования сравнивались с упомянутыми тремя работами [1-3], несмотря на то, что там изучались котельные, сжигающие ТБО, гелиоколлекторы и котлы утилизаторы, использующие бросовое тепло промышленных объектов. Вопросы компоновки котельных агрегатов особенно актуальны в свете появления технологии, предполагающей создание дополнительного тракта в котельной установке, внутри которого будет циркулировать диатермическое минеральное масло [13]. Конфигурирование генерирующего оборудования при модернизации источника опи- сывается также в [14], однако опять применительно к источнику комбинированной выработки, причем использующему в качестве топлива именно уголь, что не позволяет говорить о приемлемости сравнения полученных в [14] результатов с результатами настоящего исследования.

Контекст и специфика отечественной инфраструктуры преобразования энергии характеризуются высокой степенью аварийности систем транспортировки тепла - тепловой сети [15]. Аналогичная ситуация характерна для других стран СНГ и для всего постсоветского пространства в целом [16]. По причине значительного износа систем теплоснабжения и теплопотребляющих установок продолжают выдвигаться предложения [17] о переходе на автономное (децентрализованное) теплоснабжение, что не позволит реализовывать преимущества распределения тепловой нагрузки между тепловыми источниками и их агрегатами, а также усугубит и так негативную экологическую ситуацию [18].

Целью настоящей работы является установление оптимального с точки зрения затрат на электрическую энергию и топливо количества котельных агрегатов и их мощности применительно к трем географическим локациям. Результаты формализации и алгоритм решения должны быть пригодны для аналогичной задачи в рамках любого населенного пункта в широком диапазоне исходных данных температуры наружного воздуха, тепловой нагрузки, состава оборудования и тепловых схем источника теплоснабжения.

\section{Материалы и методы исследования}

К основным исходным данным, влияющим на выбор энергетического оборудования отопительной котельной при модернизации, относятся:

1) подключённая тепловая нагрузка и её составляющие (технология, отопление, горячее водоснабжение, вентиляция);

2) схемы подключения потребителей (зависимая, независимая, элеваторная, с насосным смешением, с регулированием теплопотребления и т. д.);

3) наличие ЦТП в схеме теплоснабжения;

4) расчётная температура наружного воздуха, продолжительность отопительного сезона, распределение температур наружного воздуха в течение отопительного сезона;

5) температурные графики системы теплоснабжения;

6) величина тепловых и гидравлических потерь в подключенной тепловой сети;

7) стоимость энергоносителей.

В зависимости от этого при реконструкции и выбирают оптимальную компоновку и тепловую схему котельной, количество и мощность котлов, параметры и число насосов и т. д. В конечном итоге основным показателем эффективности принятого решения является срок окупаемости вложенных средств. В любом случае при проведении реконструкции необходимо решать оптимизацион- 
ную задачу: выбор оптимальной тепловой схемы, типа котлов и их количество, циркуляционных, котловых насосов и другого вспомогательного оборудования.

Для решения оптимизационной задачи введём следующие обозначения.

Удельной нагрузкой на $i$-й котёл $q_{i}$ будем называть величину (1), равную отношению вырабатываемой тепловой энергии $i$-м котлом $-Q_{i}$ к номинальной мощности $i$-го котла $-Q_{i}^{\text {н }}$.

$$
q_{i}=\frac{Q_{i}}{Q_{i}^{\mathrm{H}}} .
$$

Если для уже введённых в эксплуатацию котлов зависимость КПД от удельной нагрузки определена в ходе пуско-наладочных испытаний, то для проектируемых или строящихся котлов эту зависимость можно принять усреднённой, как для котлов определённой модификации.

Формализация рассматриваемой оптимизационной задачи звучит следующим образом: средu всех возложных вариантов установки оборудования выбрать такой, при котором прялые затраты энергоносителей за отопительный сезон будут линилальныли. Это двухуровневая оптимизационная задача, для решения которой необходимо применить принцип декомпозиции. Задачу будем решать в общем виде для произвольного количества котлов. На первом уровне решим задачу оптимального распределения нагрузки между котлами разной установленной тепловой мощности при условии, что зависимость КПД котлов от вырабатываемой мощности $\eta=\eta\left(q_{i}\right)$ одинакова.

На величину $q_{i}$ наложим ограничения (2):

$$
0,3 \leq q_{i} \leq 1,1 \text {. }
$$

Суммарная выработка тепловой энергии $\mathrm{n}$ котлами (3):

$$
Q_{\Sigma}=\sum_{i=1}^{n} Q_{i} \text { МBт. }
$$

Расход газа $i$-м котлом:

$$
G_{i}=\frac{q_{i} Q_{i}^{\mathrm{H}}}{Q_{\mathrm{H}}^{p} \eta\left(q_{i}\right)} \mathrm{M}^{3} / \text { ч. }
$$

В уравнении (4) $Q_{\mathrm{H}}^{p}=33,5 \mathrm{MДж} / \mathrm{m}^{3}$ - низшая теплотворная способность природного газа.

Для принятых обозначений суммарный расход газа (5):

$$
G_{\Sigma}=\sum_{i=1}^{n} \frac{q_{i} Q_{i}^{\mathrm{H}}}{Q_{\mathrm{H}}^{p} \eta\left(q_{i}\right)} \mathbf{M}^{3} / \mathrm{u} .
$$

По условию задачи $G_{\Sigma} \rightarrow \min$. Нам надо определить, при каких значениях вектора $q_{i}$ выполняется это условие.

Применим обычный аппарат дифференциального исчисления функции нескольких переменных:

$$
\sum_{i=1}^{n} \frac{\partial G_{\Sigma}}{\partial q_{i}}=\sum_{i=1}^{n} \frac{Q_{i}^{n} \eta\left(q_{i}\right)-Q_{i}^{n} q_{i} \eta^{\prime}\left(q_{i}\right)}{Q_{p}^{\mathrm{H}} \eta^{2}\left(q_{i}\right)}=0
$$

В уравнении (6) $\eta^{\prime}\left(q_{i}\right)$ - производная от функции $\eta\left(q_{i}\right)$ при изменении удельной нагрузки $q_{i}$ на котёл.

Для выполнения равенства достаточно, чтобы каждое слагаемое равнялось нулю.

$$
\eta\left(q_{i}\right)-q_{i} \eta^{\prime}\left(q_{i}\right)=0 \text {. }
$$

Тогда оптимальная удельная нагрузка на $i$-й котёл (7):

$$
q_{i}^{\text {опт }}=\frac{\eta\left(q_{i}\right)}{\eta^{\prime}\left(q_{i}\right)},
$$

при очевидном условии: $\eta^{\prime}\left(q_{i}\right) \neq 0$.

Поскольку корреляционная зависимость $\eta\left(q_{i}\right)$ задана аналитически как непрерывная функция от $q_{i}, \eta^{\prime}\left(q_{i}\right)$ может быть также выражена аналитически.

Таким образом, при одинаковой зависимости $\eta\left(q_{i}\right)$ всех котлов, как мы приняли, для новой котельной до проведения пусконаладочных работ удельная нагрузка $q_{i}$ на все котлы при моделировании также принимается одинаковой.

В опубликованных ранее работах, например [19], показано, что наиболее рациональной, с точки зрения расхода электроэнергии, является тепловая схема, приведенная на рис. 1, поскольку для принятой тепловой схемы (качественное регулирование) отсутствуют гидравлические потери на регуляторах.

Затраты электроэнергии на перекачку теплоносителя сетевым насосом постоянны и не зависят от температуры наружного воздуха. Для расчётов принимаем температурный график $95-70{ }^{\circ} \mathrm{C}$. Выбор такого графика частично связан с применимостью полимерных трубопроводов, не подверженных коррозионному износу, при строительстве и реконструкции тепловых сетей [20].

Максимальный перепад температуры на котле $25{ }^{\circ} \mathrm{C}$. Расход теплоносителя через котёл для снятия 1,0 МВт тепловой нагрузки определим по уравнению (8):

$$
G_{\kappa}=\frac{1,0 \cdot 0,97}{4,187 \cdot 25} \cdot 3600=33,4 \mathrm{~m}^{3} / ч .
$$

КПД насосной установки $\eta_{\text {ну }}$ принимаем $76 \%$. Тогда затраты электроэнергии на перекачку теплоносителя определяем по формуле (9):

$$
P=\frac{g \rho G_{\mathrm{\kappa}} \Delta H}{1000 \eta_{\mathrm{ну}}} \kappa \mathrm{B} \mathrm{T},
$$

где $g=9,81 \mathrm{~m}^{2} / \mathrm{c}$ - ускорение свободного падения; $\rho=970$ кг $/ \mathrm{M}^{3}-$ плотность перекачиваемой жидкости; $\Delta H=2 \mathrm{~m}$ - гидравлическое сопротивление котла; $G_{\mathrm{\kappa}}-$ расход воды через котёл, $\mathrm{M}^{3} / \mathrm{c}$.

Для примера смоделируем реконструкцию котельной с подключённой тепловой нагрузкой 4,2 МВт в Республике Татарстан, Харьковской и Омской областях. Тепловые потери приняты одинаковыми и составляющими $13 \%$ от объема отпущенной тепловой энергии с коллекторов источника.

Гидравлическая схема котельной (рис. 1) подобрана следующим образом. Сетевые насосы H1, 


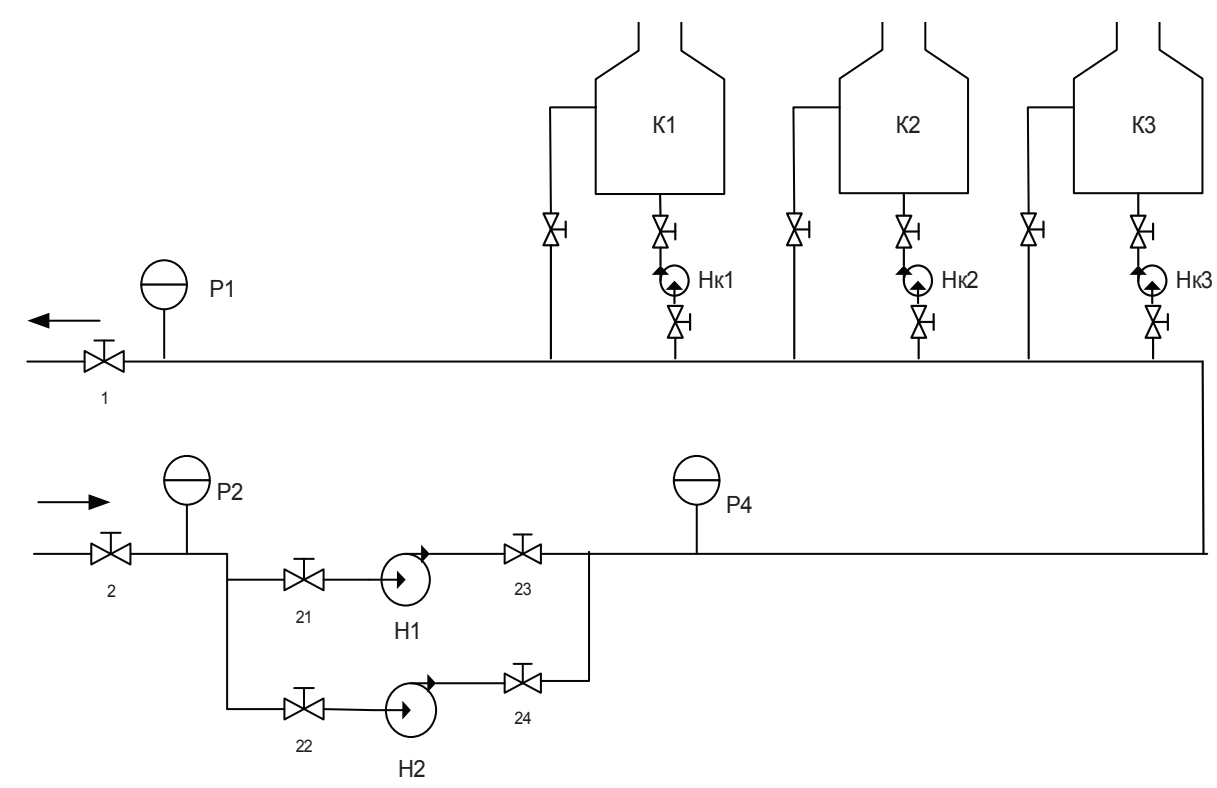

Pис. 1. Гидравлическая схела водогрейной котельной: H1, H2 - сетевые насосы (рабочий и резервный), К1, К2, К3 - отопительные котлы, Нк1, Нк2, Нк3 - котловые насосы 1-го, 2-го, 3-го котла соответственно, Р1, Р2, Р3 - манометры. Цифрами обозначена запорная арматура, стрелками - направление движения теплоносителя

Fig. 1. Scheme of central plant supplied with hot-water generators with the main district heating network pump H1, and the backup one H2; boilers $K 1, K 2$, and $K 3$; feedwater pumps $H \kappa 1, H \kappa 2$, and $H \kappa 3$; pressure sensors P1, P2, and P3. Numerals denote shut-off valves, and arrows denote direction of flow

Н2 работают только на компенсацию гидравлических потерь в тепловых сетях и создание необходимого напора перед потребителем. Гидравлическое сопротивление котла компенсирует котловой насос, который включается только при включении котла.

Как видно из рис. 1, для покрытия данной тепловой нагрузки могут быть использованы котлы мощностью $1,0,1,25 ; 1,6 ; 2 ; 2,5$ и 3,15 МВт, установленные по параллельной схеме в одной из шести нижеперечисленных комбинаций: $(2,5 \mathrm{MB}$; 2,5 МВт); (1,6 МВт; 3,15 МВт); (2,0 МВт; 3,15 МВт); (1,6 МВт; 1,6 МВт, 1,6 МВт); (2,0 МВт; 2,0 МВт, 1,0 МВт), (2,5 МВт; 1,6 МВт, 1,0 МВт). Базовый вариант - компоновка котельной двумя котлами одинаковой мощности по 2,5 МВт каждый. Для сравнения выполним расчёты для котельных, расположенных в Республике Татарстан,
Харьковской и Омской областях. Исходные данные приведены в табл. 1.

Поскольку зависимость КПД котла от удельной нагрузки на стадии проектирования и реконструкции неизвестна, для расчётов принимаем среднестатистическую зависимость КПД брутто котла от удельной нагрузки, которая приведена на рис. 2. Данная зависимость была получена автором в результате большого числа пусконаладочных работ на жаротрубных котлах разных производителей.

\section{Результаты исследования и их обсуждение}

C помощью прикладного пакета программ Statistika 6 аппроксимируем кривую, приведенную в разделе выше, полиномом пятой степени:

$$
\begin{gathered}
y=0,2564 x^{5}-1,2354 x^{4}+2,007 x^{3}- \\
-1,7134 x^{2}+0,9479 x+0,6251 .
\end{gathered}
$$

Таблица 1. Климатическая характеристика объектов исследования

\begin{tabular}{|c|c|c|c|c|c|c|c|c|c|c|c|c|}
\hline \multirow{2}{*}{$\begin{array}{c}\text { Город } \\
\text { City }\end{array}$} & \multirow{2}{*}{$\begin{array}{c}\text { Расчётная } \\
\text { температура } \\
\text { наружного воздуха } \\
\left({ }^{\circ} \mathrm{C}\right) \\
\text { Design outdoor tem- } \\
\text { perature }\left({ }^{\circ} \mathrm{C}\right)\end{array}$} & \multicolumn{10}{|c|}{$\begin{array}{c}\text { Число суток/часов за отопительный период со среднесуточными } \\
\text { температурами наружного воздуха в }{ }^{\circ} \mathrm{C} \\
\text { Duration of an average outdoor temperature through a day (days/hours, }{ }^{\circ} \mathrm{C} \text { ) }\end{array}$} & \multirow{2}{*}{$\begin{array}{c}\text { Продолжительность } \\
\text { отопительного сезона } \\
\text { (сутки/часы) } \\
\text { Duration of a heating season } \\
\text { (days/hours) }\end{array}$} \\
\hline & & 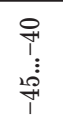 & 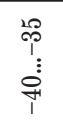 & 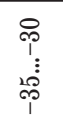 & i & 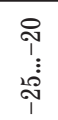 & $\underset{\substack{10 \\
1}}{\substack{1 \\
\uparrow}}$ & 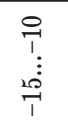 & $\begin{array}{c}1 \\
1 \\
\vdots \\
1 \\
1 \\
1 \\
0\end{array}$ & $\begin{array}{c}0 \\
\vdots \\
1 \\
1\end{array}$ & 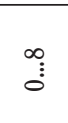 & \\
\hline \multirow{2}{*}{$\begin{array}{l}\text { Харьков } \\
\text { Kharkov }\end{array}$} & \multirow{2}{*}{-23} & - & - & - & 1,96 & 5,21 & 10 & 20 & 35 & 54 & 65 & 191 \\
\hline & & - & - & - & 47 & 125 & 246 & 487 & 829 & 1299 & 1551 & 4584 \\
\hline \multirow{2}{*}{$\begin{array}{c}\text { Казань } \\
\text { Kazan }\end{array}$} & \multirow{2}{*}{-29} & - & 0,03 & 0,82 & 3,6 & 9,2 & 19 & 31 & 40 & 55 & 59 & 217 \\
\hline & & - & 0,7 & 19,7 & 86,4 & 221 & 462 & 734 & 950 & 1318 & 1416 & 5208 \\
\hline \multirow{2}{*}{$\begin{array}{l}\text { Oмск } \\
\text { Omsk }\end{array}$} & \multirow{2}{*}{-36} & 0,23 & 2,44 & 5,4 & 12,1 & 19,5 & 30 & 34,2 & 34 & 39 & 50 & 227 \\
\hline & & 5,5 & 58,6 & 130 & 290 & 468 & 710 & 821 & 823 & 939 & 1200 & 5448 \\
\hline
\end{tabular}

Table 1. Climatic data on the tested areas 


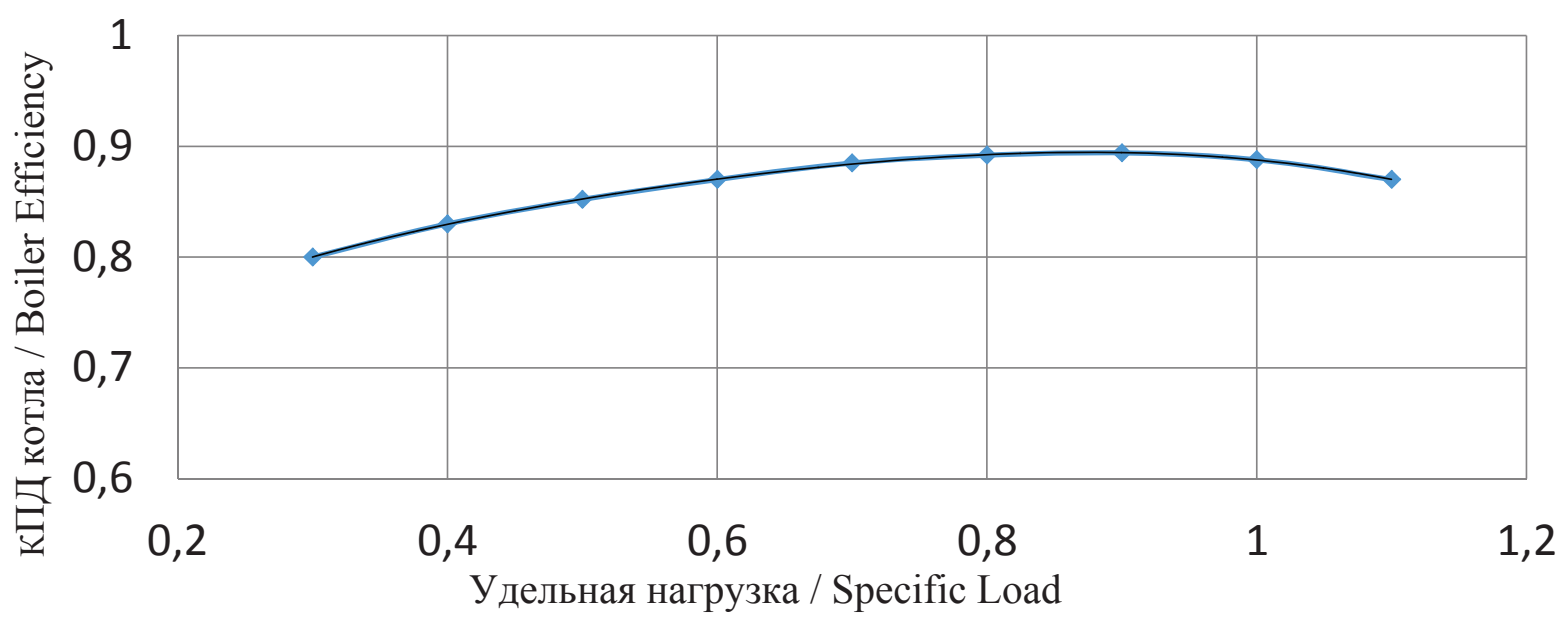

Pис. 2. Зависимость КПД котельной установки от удельной нагрузки

Fig. 2. Effect of specific load on boiler installation efficiency

Вычисление КПД по уравнению (10) при работе котла на газообразном топливе при номинальной нагрузке дает значение $92-94 \%$, что хорошо согласуется со значениями, приведенными в [12]. Результаты решения оптимизационной задачи выбора энергетического оборудования котельной для Харьковской области приведены в табл. 2.

Данные в этой и последующих таблицах, приведенные со знаком «минус», означают экономию. В [1] рассматривалось три варианта состава котлов котельной, работающей на ТБО. С точки зрения финансовых затрат выигрышным оказался вариант, согласно которому теплом от такой котельной обеспечиваются не только пригороды, но и сам г. Турин (Италия), когда общее количество потребителей достигнет 900 тыс. человек. Учитывая экологическую составляющую затрат работы котельной (налог на выбросы), при реконструкции источника теплоснабжения следует также рассматривать варианты комбинированной выработки электрической, тепловой энергии и холодоснабжения. Интересно, что в упомянутой работе [1] тариф

Таблица 2. Прямые затраты энергоносителей за сезон (натуральные и денежные показатели)

Table 2. Demand for primary energy sources during a heating season in kind and in cash

\begin{tabular}{|c|c|c|c|c|c|c|c|}
\hline Компоновка/Configuration & Ед. изм./Unit & $2,5+2,5$ & $1,6+3,15$ & $2,0+3,15$ & $1,6+1,6+1,6$ & $2,0+2,0+1,0$ & $2,5+1,6+1,0$ \\
\hline Газ/Gas & $1000 \mathrm{M}^{3}\left(\mathrm{~m}^{3}\right)$ & 1230,92 & 1213,45 & 1219,909 & 1214,06 & 1215,28 & 1208,79 \\
\hline $\begin{array}{l}\text { Отклонение } \\
\text { Difference }\end{array}$ & $1000 \mathrm{M}^{3}\left(\mathrm{~m}^{3}\right)$ & $\begin{array}{l}\text { Базовый сценарий } \\
\text { Base scenario }\end{array}$ & $-17,47$ & $-11,02$ & $-16,86$ & $-15,65$ & $-22,14$ \\
\hline $\begin{array}{l}\text { Tариф: } 10,37 \text { грн/м }{ }^{3} \\
\text { Billing price: } 10,37 \mathrm{UAH} / \mathrm{m}^{3}\end{array}$ & $\begin{array}{c}\text { тыс. грн } \\
\text { UAH } 1,000\end{array}$ & $\begin{array}{l}\text { Базовый сценарий } \\
\text { Base scenario }\end{array}$ & $-181,18$ & $-114,21$ & $-174,82$ & $-162,25$ & $-229,54$ \\
\hline Э/энергия/Electricity & $\mathrm{kBT} / \mathrm{kW}$ & 3896,38 & 3326,18 & 3568,52 & 2685,11 & 2892,87 & 3014,70 \\
\hline $\begin{array}{l}\text { Отклонение } \\
\text { Difference }\end{array}$ & $\kappa \mathrm{BT} / \mathrm{kW}$ & $\begin{array}{l}\text { Базовый сценарий } \\
\text { Base scenario }\end{array}$ & $-570,20$ & $-327,86$ & $-1211,27$ & $-1003,51$ & $-881,67$ \\
\hline $\begin{array}{l}\text { Tариф: } 2,4 \text { грн/кВтч } \\
\text { Billing price: } 2,4 \text { UAH/kWh }\end{array}$ & $\begin{array}{c}\text { тыс. грн } \\
\text { UAH } 1,000\end{array}$ & $\begin{array}{l}\text { Базовый сценарий } \\
\text { Base scenario }\end{array}$ & $-1,37$ & $-0,79$ & $-2,91$ & $-2,41$ & $-2,12$ \\
\hline $\begin{array}{l}\text { Итог } \\
\text { Total }\end{array}$ & $\begin{array}{c}\text { тыс. грн } \\
\text { UAH } 1,000\end{array}$ & $\begin{array}{l}\text { Базовый сценарий } \\
\text { Base scenario }\end{array}$ & $-182,55$ & $-115,00$ & $-177,73$ & $-164,66$ & $-231,66$ \\
\hline
\end{tabular}

Таблица 3. Прямые затраты энергоносителей за сезон (натуральные и денежные показатели)

Table 3. Demand for primary energy sources during a heating season in kind and in cash

\begin{tabular}{|c|c|c|c|c|c|c|c|}
\hline Компоновка/Configuration & Ед. изм./Unit & $2,5+2,5$ & $1,6+3,15$ & $2,0+3,15$ & $1,6+1,6+1,6$ & $2,0+2,0+1,0$ & $2,5+1,6+1,0$ \\
\hline Газ/Gas & $1000 \mathrm{M}^{3}\left(\mathrm{~m}^{3}\right)$ & 1272,08 & 1252,06 & 1247,383 & 1252,67 & 1236,51 & 1241,95 \\
\hline $\begin{array}{l}\text { Отклонение } \\
\text { Difference } \\
\end{array}$ & $1000 \mathrm{~m}^{3}\left(\mathrm{~m}^{3}\right)$ & $\begin{array}{l}\text { Базовый сценарий } \\
\text { Base scenario }\end{array}$ & $-20,02$ & $-24,70$ & $-19,41$ & $-35,57$ & $-30,13$ \\
\hline $\begin{array}{l}\text { Tариф: } 5,48 \mathrm{p} . / \mathrm{m}^{3} \\
\text { Billing price: } 5,48 \text { roubles per } \mathrm{m}^{3}\end{array}$ & $\begin{array}{c}\text { тыс. } p . \\
1,000 \text { roubles }\end{array}$ & $\begin{array}{l}\text { Базовый сценарий } \\
\text { Base scenario }\end{array}$ & $-109,7$ & $-135,3$ & $-106,4$ & $-194,9$ & $-165,1$ \\
\hline Э/энергия/Electricity & $\kappa \mathrm{B} \mathrm{T} / \mathrm{kW}$ & 3413,9 & 3634,8 & 3488,3 & 3578,8 & 3051,4 & 2957,4 \\
\hline \begin{tabular}{|l|} 
Отклонение \\
Difference \\
\end{tabular} & кВт $/ \mathrm{kW}$ & $\begin{array}{l}\text { Базовый сценарий } \\
\text { Base scenario }\end{array}$ & 220,9 & 74,4 & 164,9 & $-362,4$ & $-456,5$ \\
\hline $\begin{array}{l}\text { Tариф: } 3,56 \mathrm{p} . / \mathrm{\kappa} \text { Ттч } \\
\text { Billing price: } 3,56 \text { roubles per } \mathrm{kWh}\end{array}$ & $\begin{array}{c}\text { тыс. } p . \\
1,000 \text { roubles }\end{array}$ & $\begin{array}{l}\text { Базовый сценарий } \\
\text { Base scenario }\end{array}$ & 0,8 & 0,3 & 0,6 & $-1,3$ & $-1,6$ \\
\hline $\begin{array}{l}\text { Итог } \\
\text { Total }\end{array}$ & $\begin{array}{c}\text { тыс. } p . \\
1,000 \text { roubles }\end{array}$ & $\begin{array}{l}\text { Базовый сценарий } \\
\text { Base scenario }\end{array}$ & $-108,9$ & 135,1 & $-105,8$ & $-196,2$ & $-166,7$ \\
\hline
\end{tabular}


Таблица 4. Прялые затраты энергоносителей за сезон (натуральные и денежные показатели)

Table 4. Demand for primary energy sources during a heating season in kind and in cash

\begin{tabular}{|c|c|c|c|c|c|c|c|}
\hline Компоновка/Configuration & Ед. изм./Unit & $2,5+2,5$ & $1,6+3,15$ & $2,0+3,15$ & $1,6+1,6+1,6$ & $2,0+2,0+1,0$ & $2,5+1,6+1,0$ \\
\hline Газ/Gas & $1000 \mathrm{M}^{3}\left(\mathrm{~m}^{3}\right)$ & 1257,57 & 1252,64 & 1252,108 & 1262,22 & 1239,81 & 1238,61 \\
\hline $\begin{array}{l}\text { Отклонение } \\
\text { Difference }\end{array}$ & $1000 \mathrm{M}^{3}\left(\mathrm{~m}^{3}\right)$ & $\begin{array}{l}\text { Базовый сценарий } \\
\text { Base scenario }\end{array}$ & $-4,93$ & $-5,46$ & 4,64 & $-17,77$ & $-18,96$ \\
\hline $\begin{array}{l}\text { Тариф: } 4,93 \mathrm{p} . / \mathrm{M}^{3} \text { Billing price: } \\
4,93 \text { roubles per } \mathrm{m}^{3}\end{array}$ & $\begin{array}{c}\text { тыс. } p . \\
1,000 \text { roubles }\end{array}$ & $\begin{array}{l}\text { Базовый сценарий } \\
\text { Base scenario }\end{array}$ & $-24,3$ & $-26,9$ & 22,9 & $-87,6$ & $-93,5$ \\
\hline Э/энергия/Electricity & $\kappa \mathrm{B} \mathrm{T} / \mathrm{kW}$ & 3947,0 & 3791,2 & 4096,2 & 4112,3 & 3746,5 & 3061,6 \\
\hline $\begin{array}{l}\text { Отклонение } \\
\text { Difference }\end{array}$ & кВт $/ \mathrm{kW}$ & $\begin{array}{l}\text { Базовый сценарий } \\
\text { Base scenario }\end{array}$ & $-155,8$ & 149,2 & 165,3 & $-200,5$ & $-885,4$ \\
\hline $\begin{array}{l}\text { Тариф: } 3,68 \text { p. } / \kappa B \text { тч Billing price: } \\
3,68 \text { roubles per kWh }\end{array}$ & $\begin{array}{c}\text { тыс. } p . \\
1,000 \text { roubles }\end{array}$ & $\begin{array}{l}\text { Базовый сценарий } \\
\text { Base scenario }\end{array}$ & $-0,6$ & 0,5 & 0,6 & $-0,7$ & $-3,3$ \\
\hline Итог/Total & $\begin{array}{c}\text { тыс. } p . \\
1,000 \text { roubles }\end{array}$ & $\begin{array}{l}\text { Базовый сценарий } \\
\text { Base scenario }\end{array}$ & $-24,9$ & $-26,4$ & 23,5 & $-88,3$ & $-96,7$ \\
\hline
\end{tabular}

на электрическую энергию установлен в размере $74,72 € / \mathrm{MWh}$, а на тепловую - $20 € / \mathrm{MWh}$, т. е. сопоставимый относительно рассмотренных населенных пунктов России и Украины. Результаты решения оптимизационной задачи выбора энергетического оборудования котельной для Республики Татарстан показаны в табл. 3.

Результаты решения оптимизационной задачи выбора энергетического оборудования котельной для Омской области приведены в табл. 4.

Для сравнения, в работе [9] получена гораздо более ощутимая экономия в размере 22,37 млн р. в год, однако предложенные там мероприятия по оборудованию водогрейной котельной собственным источником электрической генерации требуют масштабных инвестиционных затрат в размере $65 \mathrm{млн}$ р. и не связаны с простым конфигурированием оборудования котельной, как в настоящей работе.

\section{Заключение}

Как показало решение оптимизационной задачи, в разных регионах компоновка котельной одной и той же мощности должна быть различная. Для Харьковской $\left(T_{p}=-23^{\circ} \mathrm{C}\right)$ и Омской $\left(T_{p}=-36{ }^{\circ} \mathrm{C}\right)$ областей оптимальным является состав оборудования 2,$5 ; 1,6 ; 1,0$ МВт, а для г. Казани $\left(T_{p}=-29^{\circ} \mathrm{C}\right)-2,0$; 2,$0 ; 1,0$ МВт. Традиционный состав оборудования $2,5+2,5$ МВт является максимально убыточным с точки зрения прямых затрат для Харьковской области и Республики Татарстан. Для г. Омска же наиболее нерациональным будет котельная в составе трёх котлов одинаковой мощности 1,6 МВт.

\section{СПИСОК ЛИТЕРАТУРЫ}

1. Panepinto D., Zanetti M.C. Municipal solid waste incineration plant: a multi-step approach to the evaluation of an energy-recovery configuration // Waste Management. - 2018. - V. 73. P. 332-341. D0I: 10.1016/j.wasman.2017.07.036

2. Innovative planning and evaluation system for district heating using waste heat considering spatial configuration: a case in Fukushima, Japan / Y. Dou, T. Togawa, L. Dong, M. Fujii, S. Ohnishi, H. Tanikawa, T. Fujita // Resources, Conservation and Recycling. - 2018. - V. 128. - P. 406-416. DOI: 10.1016/j.resconrec.2016.03.006

3. Photovoltaic thermal hybrid solar collector and district heating configurations for a Central European multi-family house /
Использованный в настоящей работе метод решения двухуровневой оптимизационной задачи, для решения которой применялся принцип декомпозиции, позволил установить оптимальную из шести возможных комбинаций компоновку оборудования. В частности, показана возможность производства при минимальных затратах первичных энергоносителей, что, в свою очередь, приведет к снижению вредного воздействия на окружающую среду и позволит говорить об энергоэффективной технологии производства и преобразования энергии на основе георесурсов. Результаты в форме денежных показателей указывают на то, что приведенный математический аппарат может быть полезен и пригоден для решения аналогичной задачи в рамках любого населенного пункта с учетом разности тарифов на газ и электрическую энергию. Дальнейшее развитие настоящего исследования предполагается выполнить путем рассмотрения влияния появления дополнительного циркуляционного контура с теплоносителем из минерального масла [13] на оптимальный состав оборудования котельной.

Работа выполнена при финансовой поддержке стипендии Правительства Российской Федерации (приказ Минобрнауки № 860 от 29.08.2017). Авторы также выражают признательность коллективу кафедры теплотехники и энергоэффективных технологий Национального технического университета «Харьковский Политехнический институт» (заведующий кафедрой д.т.н., проф. Ганжа А.Н.) за предоставленные данные и постоянную помощь в написании работы.

N. Pardo García, G. Zubi, G. Pasaoglu, R. Dufo-López / / Energy Conversion and Management. - 2017. - V. 148. - P. 915-924. DOI: $10.1016 / j$.enconman.2017.05.065

4. Chicherin S.V. Comparison of a district heating system operation based on actual data - Omsk city, Russia, case study // International Journal of Sustainable Energy. - 2019. - V 38. - № 6. P. 603-614. DOI: $10.1080 / 14786451.2018 .1548466$

5. Terhan M., Comakli K. Energy and exergy analyses of natural gas-fired boilers in a district heating system // Applied Thermal Engineering. - 2017. - V. 121. - P. 380-387. D0I: 10.1016/j.applthermaleng.2017.04.091

6. Fang T., Lahdelma R. Genetic optimization of multi-plant heat production in district heating networks // Applied Energy. - 
2015. - V. 159. - P. 610-619. DOI: 10.1016/j.apenergy.2015.09.027

7. Franco A., Versace M. Optimum sizing and operational strategy of CHP plant for district heating based on the use of composite indicators // Energy. - 2017. - V. 124. - P. 258-271. D0I: 10.1016/j.energy.2017.02.062

8. Джангиров В.А., Лелюшкин Н.В., Маслов В.В. Кризис теплофикации? Выход есть! // Энергетик. - 2018. - № 1. - С. 3-8.

9. Энергосберегающая технология модернизации квартальных тепловых станций с устройством автономных низкопотенциальных электрогенерирующих установок / П.П. Безруких, Ф.А. Поливода, А.А. Дзюба, В.П. Щербаков, Л.А. Шатров, Т.И. Набатчикова // Энергобезопасность и энергосбережение. - 2017. - № 6. - С. 19-28.

10. Tańczuk M., Skorek J., Bargiel P. Energy and economic optimization of the repowering of coal-fired municipal district heating source by a gas turbine // Energy Conversion and Management. 2017. - V. 149. - P. 885-895. DOI: 10.1016/j.enconman.2017.03.053

11. Хаустов С.А., Хаустова О.В., Ермолаев А.Н. Динамическая модель твердотопливного отопительного котла // Известия Томского политехнического университета. Инжиниринг георесурсов. - 2018. - Т. 329. - № 2. - С. 16-26.

12. Тринченко А.А., Парамонов А.П. Техническое перевооружение котла БКЗ-210-140-13,8 на основе низкотемпературной вихревой технологии сжигания // Научно-технические ведомости СПбПУ. Естественные и инженерные науки. - 2017. T. 23. - № 2. - C. 62-74. DOI: 10.18721/JEST.230206

13. Из опыта реконструкции производственной теплогенерирующей установки с использованием котлов на отработанном ма- сле / Т.И. Королева, Н.В. Аржаева, О.В. Тараканов, Н.Ю. Иващенко / Региональная архитектура и строительство. - 2017. № 1 (30). - C. 151-158.

14. Балтян В.Н., Ефимов Н.Н., Цхяев А.Д. К вопросу модернизации котельного оборудования угольных ТЭС // Известия высших учебных заведений. Северо-Кавказский регион. Серия: Технические науки. - 2017. - № 1 (193). - С. 50-53.

15. Збараз Л.И., Чичерин С.В. Методика оценки ущерба от аварийной ситуации в системе теплоснабжения // Науковий вісник будівництва. - 2017. - № 4 (90). - С. 218-224.

16. Абдыкалыков Э.Э., Батаканов М.Т. Теплофикация г. Бишкек // Известия Кыргызского государственного технического университета им. И. Раззакова. - 2017. - Т. 44. - № 4. - С. 14-20.

17. Новосельцев Б.П. Некоторые аспекты улучшения качества теплоснабжения зданий // Жилищное хозяйство и коммунальная инфраструктура. - 2017. - № 3 (2). - С. 22-26.

18. К вопросу об эффективности и экологической безопасности теплоэнергетических систем / А.Н. Егоров, Е.В. Криволапова, Н.В. Бутримова, А.А. Егоров // Вестник Оренбургского государственного университета. - 2017. - № 12 (212). - С. 94-97.

19. Збараз Л.И. Моделирование децентрализованного источника теплоснабжения и выбор оптимальных параметров его работы // Коммунальное хозяйство городов. - 2015. - № 123. - С. 91-97.

20. Chicherin S., Volkova A., Latõšov E. GIS-based optimisation for district heating network planning // Energy Procedia. - 2018. V. 149. - P. 635-641. D0I: 10.1016/j.egypro.2018.07.107

Поступила 11.09.2018 г.

\section{Информация об авторах}

Збараз Л.И., кандидат технических наук, доцент кафедры теплотехники и энергоэффективных технологий Национального технического университета «Харьковский Политехнический институт».

Чичерин C.B., аспирант кафедры теплоэнергетики Омского государственного университета путей сообщения. 
UDC 662.71.74

\title{
CONFIGURING A DISTRICT HEATING PLANT UNDER RECONSTRUCTION: OPTIMUM QUANTITY OF BOILER UNITS
}

\author{
Leonid I. Zbaraz', \\ Zbaraz_Len@ukr.net \\ Stanislav V. Chicherin², \\ man_csv@hotmail.com \\ 1 National Technical University «Kharkiv Polytechnic Institute», \\ 2, Kyrpychova str., 61002, Kharkiv, Ukraine. \\ 2 Omsk State Transport University, \\ 35, Marx avenue, Omsk, 644046, Russia.
}

Relevance. An over-dimensioning district heating plant is costly, as there will be more losses during low-load periods and more capital bound in the investments. During the highload (peak load) periods, a regular heat plant might not be able to deliver enough heat to the system. To ensure that the customers still receive their heat, the peak production plants are often created to top-up during peak-load periods. As these peak-load plants are often small in size and constructed only to run for short periods of time, they are often fossil-fuel fired, or fired by other highly refined, and hence expensive fuels.

The aim of this work is to develop a tool (a multi-step approach) capable of adjusting the secondary load to match the optimum load structure to save primary energy sources, and meanwhile ensure the demand and supply balance.

Methods. The optimum scheme is selected by evaluating and comparing several schemes, considering the constraints from the original system configuration, which results in a limited number of independent variables. We dealt with sequencing control for multiple boilers as required by load demand that is desirable when two or more boilers are installed in parallel. This can be accomplished by utilizing a steam flowmeter or water temperature sensor, when applicable, to energize an additional boiler when the load cannot be met by the boilers already on-line. The difference in the efficiency between boilers located in different places can be appreciable if the fuel input to a boiler is modulated. This increase in efficiency is due to the increase in the ratio of heat exchanger surface area to heat input as the firing rate is reduced. The mathematical model should be solved with a programming software, such as Statistica 6.

Results. Three different geographical locations were considered. The authors have calculated the mean annual values for electricity accounted 3,68 roubles per kWh, and values for the days of highest plant activity accounted 5,48 roubles per cubic meter of gas, for comparison with an acceptable concentration level for the exposed population. The results indicate that for thermal energy value varying under six scenarios (from 2,5+2,5 to 2,5+1,6+1,0 MW configuration) the situation is completely different when implementing the optimization model for Kazan and Kharkiv. It can be also seen that 2,5+1,6+1,0 MW configuration (and in particular Kharkiv and Omsk: related to the warmest and coldest locations respectively) is more advantageous from the economic point of view.

Conclusions. In this work a multi-step approach was used to evaluate the economic aspects of six heat-only boiler plant energy-recovery configurations. The aim of the developed methodology was to supply useful information to a designer. The obtained results were interesting, and they can certainly be justified in practice. In particular, the results showed a low energy-production cost for production of heat, as well as an opportunity to minimize environmental impacts. The economic analysis results, however, show that the proposed method is quite helpful, because of the considering price difference between gas and electrical energy distributions.

Key words:

Primary energy, district heating, natural gas, boiler, production, generation, optimization, minimization, costs.

This research was supported by the Government of the Russian Federation under Project No. 860 (Decree dated August, 8 2017). We thank the scholars of the National Technical University «Kharkiv Polytechnic Institute», namely Professor, Dr. Sc. Anton Ganzha for the supplied data and for continuous support in the research processing.

\section{REFERENCES}

1. Panepinto D., Zanetti M. C. Municipal solid waste incineration plant: a multi-step approach to the evaluation of an energy-recovery configuration. Waste Management, 2018, vol. 73, pp. 332-341. DOI: $10.1016 /$ j.wasman.2017.07.036

2. Dou Y., Togawa T., Dong L., Fujii M., Ohnishi S., Tanikawa H., Fujita T. Innovative planning and evaluation system for district heating using waste heat considering spatial configuration: a case in Fukushima, Japan. Resources, Conservation and Recycling, 2018, vol. 128, pp. 406-416. DOI: 10.1016/j.resconrec.2016.03.006

3. Pardo García N., Zubi G., Pasaoglu G., Dufo-López R. Photovoltaic thermal hybrid solar collector and district heating configurations for a Central European multi-family house. Energy Conversion and Management, 2017, vol. 148, pp. 915-924. DOI: 10.1016/j.enconman.2017.05.065
4. Chicherin S. V. Comparison of a district heating system operation based on actual data - Omsk city, Russia, case study. International Journal of Sustainable Energy, 2019, vol. 38 no. 6, pp. 603-614. D0I: $10.1080 / 14786451.2018 .1548466$

5. Terhan M., Comakli K. Energy and exergy analyses of natural gas-fired boilers in a district heating system. Applied Thermal Engineering, 2017, vol. 121, pp. 380-387. DOI: 10.1016/j.applthermaleng.2017.04.091

6. Fang T., Lahdelma R. Genetic optimization of multi-plant heat production in district heating networks. Applied Energy, 2015, vol. 159 , pp. 610-619. D0I: 10.1016/j.apenergy.2015.09.027

7. Franco A., Versace M. Optimum sizing and operational strategy of CHP plant for district heating based on the use of composite indicators. Energy, 2017, vol. 124, pp. 258-271. DOI: 10.1016/j.energy.2017.02.062 
8. Dzhangirov V.A., Lelyushkin N.V., Maslov V.V. Crisis of district heating? There is the way out! Energetik, 2018, no. 1, pp. 3-8. In Rus.

9. Bezrukikh P., Polivoda F., Dzyuba A., Scherbakov V., Shatrov L., Nabatchikova T. Energy saving reconstruction of district heating stations with independent low-potential power generation unit. Energy Safety and Energy Economy, 2017, vol. 6, pp. 19-28. In Rus. DOI: 10.18635/2071-2219-2017-6-19-28

10. Tańczuk M., Skorek J., Bargiel P. Energy and economic optimization of the repowering of coal-fired municipal district heating source by a gas turbine. Energy Conversion and Management, 2017, vol. 149, pp. 885-895. DOI: 10.1016/j.enconman.2017.03.053

11. Khaustov S.A. Computational model of a solid fuel heating boiler. Bulletin of the Tomsk Polytechnic University. Geo Assets Engineering, 2018, vol. 329, no. 2, pp. 16-26. In Rus.

12. Trinchenko A.A., Paramonov A.P. Technical re-equipment of the BKZ-210-140-13,8 boiler on the basis of low-temperature vortex combustion technology. St. Petersburg Polytechnic University Journal of Engineering Science and Technology, 2017, vol. 23, no. 2, pp. 62-74. In Rus. DOI: 10.18721/JEST.230206

13. Koroleva T.I., Arzhaeva N.V., Tarakanov 0.V., Ivashchenko N.U. From the experience of reconstruction of industrial heat transfer installation using boilers on waste oil. Regional architecture and engineering, 2017, no. 1 (30), pp. 151-158. In Rus.

14. Baltyan V.N., Efimov N.N., Tskhyaev A.D. To the problem of boiler equipment coal plants modernization. University News North-
Caucasian Region Technical Sciences Series, 2017, no. 1 (193), pp. 50-53. In Rus.

15. Zbaraz L.I., Chicherin S.V. Methodology of estimation of damage from emergency situation in the heat supply system. Scientific Bulletin of Civil Engineering, 2017, vol. 4, no. 90, pp. 218-224. In Rus.

16. Abdykalykov E., Batakanov M. Bishkek city district heating process [Teplofikatsiya g. Bishkek]. Herald of Kyrgyz State Technical University named after I. Razzakov, 2017, vol. 44, no. 4, pp. 14-20.

17. Novoseltsev B.P. Some aspects of improving the quality of heat supply of buildings. Housing and communal infrastructure, 2017, no. 3 (2), pp. 22-26. In Rus.

18. Egorov A.N., Krivolapova E.V., Butrimova N.V., Egorov A.A. To the issue of efficiency and ecological safety of heat power systems. Herald of Orenburg State University, 2017, vol. 212, no. 12 , pp. 94-97. In Rus.

19. Zbaraz L.I. Modelling of the decentralized source of the heat supply and choice of optimum parameters of its work. Municipal economy of cities, 2015, no. 123, pp. 91-97. In Rus.

20. Chicherin S., Volkova A., Latõšov E. GIS-based optimisation for district heating network planning. Energy Procedia, 2018, vol. 149 , pp. $635-641$. D0I: $10.1016 /$ j.egypro.2018.07.107

Received: 11 September 2018.

\section{Information about the authors}

Leonid I. Zbaraz, Cand. Sc., associate professor, National Technical University «Kharkiv Polytechnic Institute».

Stanislav V.Chicherin, postgraduate student, Omsk State Transport University. 